\title{
Based on Transcriptome Sequencing: Clinicians Need to Pay Attention to Fever Caused by Common Respiratory Pathogens During the Global Outbreak of COVID-19
}

\section{Sheng Yin}

Second Xiangya Hospital Central South University

\section{Zeyou Wang}

Second Xiangya Hospital Central South University

Min Wang ( $\nabla$ wangmin0000@csu.edu.cn)

Second Xiangya Hospital Central South University https://orcid.org/0000-0002-3420-4031

\section{Wenlong Wang}

Second Xiangya Hospital Central South University

\section{Research}

Keywords: COVID-19, SARS-CoV-2, Hunan, China, Respiratory Pathogens

Posted Date: July 2nd, 2021

DOI: https://doi.org/10.21203/rs.3.rs-490705/v2

License: (c) (1) This work is licensed under a Creative Commons Attribution 4.0 International License.

Read Full License 


\section{Abstract}

\section{Objectives:}

During the COVID-19 pandemic, clinicians and public health decision-makers especially focus on fever patients. Other common pathogens that may cause fever are easily overlooked. We aimed to describe the pathogen infection and epidemic trend of non-SARS-CoV-2 occurring in hospitalized patients.

\section{Methods:}

An observational cohort study of 733 consecutive patients admitted to Hospital Clinic of the Second Xiangya Hospital for COVID-19. All samples of a pharyngeal swab from patients with fever have been tested for nucleic acid and immune antigens of SARS-CoV-2 and Influenza A/B virus. 649 fever patients have been tested for nucleic acid in ten respiratory pathogens. Macrotranscriptome sequencing was performed on 26 samples.

\section{Results:}

Of a total of 733 patients with fever, $2.05 \%$ patients had confirmed SARS-CoV-2 infections. Fever patients with common respiratory pathogens in fever patients was $8.78 \%$. There is no integration phenomenon between SARS-Cov-2 and the human genome. SARS-CoV-2 positive samples will also be infected with other viruses, especially adenovirus. Macrotranscript analysis showed that there was no significant difference in the species and genus levels of pathogens between Covid-19 patients and other fever patients. The main pathways that affect human metabolism after SARS-Cov-2 infection are the CalvinBenson-Bassham cycle, pyrimidine deoxyribonucleotides de novo biosynthesis I and D-galactose degradation V.

\section{Conclusions:}

Most patients have a fever caused by common respiratory pathogens. Clinicians still need to pay more attention to infections of common respiratory pathogens in addition to SARS-CoV-2. China's public health measures to stop the spread of the epidemic have proven effective.

\section{Background}

In December 2019, a series of acute respiratory diseases occurred in Wuhan, Hubei Province, China, now called Coronavirus Disease 2019 (COVID-19) ${ }^{1}$. The disease has rapidly spread from Wuhan to other regions. As of February 24, 2021, The number of confirmed COVID-19 cases worldwide is 112,534,427. A new type of coronavirus (SARS-CoV-2) was identified in a bronchial alveolar lavage fluid sample from a patient in Wuhan City and confirmed it as the cause of COVID- $19^{2}$. Now the virus is renamed SARS-CoV-2 by $\mathrm{WHO}^{3}$. Whole-genome sequencing and phylogenetic analysis indicate that SARS-CoV-2 is a unique clade of $\beta$-coronal viruses associated with human severe acute respiratory syndrome (SARS) and the 
Middle East respiratory syndrome (MERS) ${ }^{2}$. Like other beta coronaviruses, the genome of SARS-CoV-2 has a long ORF1 1 b polyprotein at the $5^{\prime}$ end, followed by four major structural proteins, including the spike surface glycoprotein, small envelope protein, matrix protein, and nucleocapsid protein ${ }^{4}$. SARS-CoV-2 is very similar to bat coronavirus, and bats are presumed to be the main source and bats are presumed to be the main source ${ }^{5}$. Although the origin of SARS-CoV-2 is still under investigation, current evidence suggests that it spread to humans through the spread of wild animals illegally sold in the South China ${ }^{6}$.

Huang et al. first reported 41 COVID-19 cases, most of whom had a history of exposure to the South China Seafood Wholesale Market. The patient's clinical manifestations included fever, unproductive cough, dyspnea, myalgia, fatigue, normal or decreased white blood cell count, and imaging evidence of pneumonia ${ }^{7}$. However, only a small number of articles reported co-infection in patients with COVID-198,9. Co-infection at COVID-19 diagnosis is uncommon. Few patients developed superinfections during hospitalization. Patients with fever often detect ten pathogens. Influenza A/B virus are common influenza virus. They are mainly prone to mutation and the Influenza $A$ virus main subtype is called avian influenza. Symptoms after infection are mainly high fever and cough, accompanied by pneumonia and small airway dysfunction ${ }^{10}$. The four subtypes of human parainfluenza viruses have different clinical and epidemiological characteristics. The most typical clinical feature of type I and type II is that it causes laryngotracheobronchitis in children, type I is the main cause of this type of laryngotracheobronchitis in children, and type II is the second. Both types I and II can cause other upper and lower respiratory tract diseases. Type III often causes pneumonia and bronchiolitis ${ }^{11}$. Human metapneumovirus (hMPV) is a single-stranded ribonucleic acid virus in the Paramyxoviridae family and was first discovered in the Netherlands in 2001. The virus mainly causes acute respiratory infections in children. Symptoms include fever, cough, shortness of breath, and difficulty breathing. Adults with weak resistance also have a chance to be infected ${ }^{12}$. Human Boca virus (hBoV) is a genus of the Parvovirinae of the Parvoviridae family. Boca virus usually infects the intestines and respiratory tract. Some viruses may be transmitted through the placenta and cause congenital infection of the fetus. Human Boca virus spreads through the air, and it is easy for infants and young children to suffer from diseases such as pneumonia, bronchitis, and bronchopneumonia ${ }^{13}$. Acute infectious diseases caused by Adenovirus can easily invade the mucosa of the respiratory tract and digestive tract, conjunctiva, urinary tract, and lymph nodes. The main manifestations are acute upper respiratory tract infections (acute respiratory infections are caused by Adenovirus accounting for 2-4\%), followed by eye and gastrointestinal infections. Approximately half of the patients have a recessive infection. Infants and young children are susceptible to Adenovirus pneumonia, with severe illness and high mortality ${ }^{14}$. Coronavirus is a single-stranded positive-stranded RNA with a diameter of about 100 nanometers, which is irregularly round or quasi-circular. Under the electron microscope, the shape is crown-shaped. It belongs to the coronavirus family. The human coronavirus (hCoV) has two antigen types, OC43 and 229E. The incubation period is generally 2 to 5 days. The typical manifestation is the common cold, with a blocked nose, runny nose, sore throat, red throat, and general malaise. Pneumonia may occasionally occur and cause asthma in children. The OC43 strain causes more severe symptoms than $229 \mathrm{E}^{15}$. Respiratory syncytial virus (RSV) is an RNA virus 
belonging to the Paramyxoviridae family. The disease is spread by air droplets and close contact. Infants and young children have severe symptoms, including high fever, rhinitis, pharyngitis, and laryngitis, and later manifested as bronchiolitis and pneumonia. A small number of sick children may be complicated by otitis media, pleurisy, and myocarditis. After adults and older children are infected, the main manifestations are upper respiratory tract infections ${ }^{16}$. The pathological changes of Mycoplasma pneumoniae (MP) are mainly interstitial pneumonia, sometimes complicated by bronchial pneumonia, called primary atypical pneumonia. If they have, they are just general respiratory symptoms such as headache, sore throat, fever, and cough, but there are also individual deaths ${ }^{17}$. During the COVID-19 pandemic, clinicians and public health decision-makers especially focus on fever patients. Other common pathogens that may cause fever are easily overlooked ${ }^{18,19}$. Doing so is likely to cause a waste of medical resources, delays in medical treatment, and social panic. The purpose of this case series is to describe the pathogen infection and epidemic trend of non-SARS-CoV-2 occurring in hospitalized patients with COVID-19.

\section{Methods}

\section{Sample Collection}

This study was approved by the Hospital Ethics Committee, and all study participants were informed. The study population consisted of 733 fever patients at the Second Xiangya Hospital of Central South University, Hunan province, China, between January to February 2020, aged 20 to 70 years. All the patients were diagnosed by two experienced doctors. The samples from the participants were obtained before clinical intensive treatment. Participants who received preoperative radiotherapy or chemotherapy were excluded. Respiratory and stool samples were collected with the consent of all patients. Unless otherwise stated, patients were sampled without gender or age bias. Swabs and stool were stored at $-20^{\circ} \mathrm{C}$ until use.

\section{RNA extraction and qRT-PCR}

Whenever a commercial kit is used, the manufacturer's instructions should be followed without modification. Nucleic acids were extracted by SSNP-2000A Automatic Nucleic Acid Extraction Instrument and nucleic acid extraction kits (Sansure biotech, China). $20 \mu \mathrm{L}$ extracted nucleic acid sample was added into a PCR amplification tube which contains $30 \mu \mathrm{L}$ of the prepared PCR-mixed solution, and perform fluorescence quantitative PCR detection on a fluorescent PCR instrument ( detected through fluorescence quantitative PCR using a fluorescent PCR instrument.) Amplification was performed as follows: $50^{\circ} \mathrm{C}$ for 30 minutes, $95^{\circ} \mathrm{C}$ for 60 seconds, and then 40 cycles, including $95^{\circ} \mathrm{C}$ for 15 seconds, $60^{\circ} \mathrm{C}$ for 30 seconds.

The primer and probe sequences ${ }^{20}$ :

ORF1b gene 
5'-TGGGGYTTTACRGGTAACCT-3' (Forward)

5'-AACRCGCTTAACAAAGCACTC-3' (Reverse)

$\mathrm{N}$ gene

5'-TAATCAGACAAGGAACTGATTA-3' (Forward)

5'-CGAAGGTGTGACTTCCATG-3' (Reverse)

Nucleic acids were analysised Influenza $A / B$ virus nucleic acids test kits (bioPerfectus, China). $5 \mu \mathrm{L}$ extracted nucleic acid sample was added into a PCR amplification tube which contains $20 \mu \mathrm{L}$ of the prepared PCR-mixed solution and performs fluorescence quantitative PCR detection on a fluorescent PCR instrument. Amplification was performed as follows: $50^{\circ} \mathrm{C}$ for 30 minutes, $95^{\circ} \mathrm{C}$ for 5 minutes, and then 40 cycles, including $95^{\circ} \mathrm{C}$ for 10 seconds, $55^{\circ} \mathrm{C}$ for 40 seconds. The reference value of the Influenza $A$ virus is $34.7(\mathrm{CT})$. The reference value of the Influenza $B$ virus is $34.8(\mathrm{CT})$.

\section{Colloidal gold immunochromatography}

The nurse uses a throat swab to apply on both sides of the back wall of the throat or in the nasal cavity. Take a sufficient amount of specimens and put them in a special disposable specimen collection tube for inspection. The specimens should be sent for inspection immediately after collection or stored at $4^{\circ} \mathrm{C}$ for no more than 3 days. Otherwise, it should be stored below $-20^{\circ} \mathrm{C}$, and repeated freezing and thawing should not exceed 3 times. According to the Influenza A/B virus antigen detection instructions (Wondfo biotech, China), drop 8 drops of the dilution liquid in the dropper bottle into the plastic tube of the kit, add a sufficient amount of the sample, stir and repeatedly squeeze the tube wall, so that the sample is fully dissolved to the dilution In the liquid. Use a pipette to slowly add 3-4 drops (about 100ul) of the sample solution into the sample hole of the test card, and start recording the time.

\section{Ten respiratory pathogen nucleic acid detection (multiple melting curve method)}

The throat swab samples were sucked into the supernatant according to the instructions of the "Nucleic Acid Extraction or Purification Reagents", and the nucleic acid was extracted according to the instructions of the EX-DNA/RNA Virus Nucleic Acid Extraction Kit (Tianlong, China). Configure the RT-PCR system, add 4.4 $\mu \mathrm{LRes}$ reaction solution, and $0.6 \mu \mathrm{LRes}$ enzyme solution to a $1.5 \mathrm{~mL}$ centrifuge tube in turn. Add $25 \mu \mathrm{L}$ of mineral oil after centrifugation. Place the positive control, negative control and samples on the PCR machine for amplification. Amplification was performed as follows: $50^{\circ} \mathrm{C}$ for 30 minutes, $95^{\circ} \mathrm{C}$ for 15 minutes, and then 40 cycles, including $94^{\circ} \mathrm{C}$ for 30 seconds, $55^{\circ} \mathrm{C}$ for 30 seconds, $72^{\circ} \mathrm{C}$ for 30 seconds. After the program is run, the instrument will automatically pay to check the Tm value.

\section{Data collection}

We reviewed the care records and laboratory test results of fever patients. Admission data for these patients were from January 26th to February 1st, 2020. The two researchers also independently reviewed 
data collection forms to confirm repeatedly.

\section{Bioinformatics analysis process}

The use of different methodological kits to detect SARS-CoV-2 and other common respiratory viruses on samples of fever patients limits our further understanding of SARS-CoV-2 co-infection. We commissioned Sansure to perform RNA metagenomic sequencing on 26 fever patients' samples (SARS-CoV-2 positive: $n=16$, SARS-CoV-2 negative: $n=10$ throat swab: $n=22$, stool: $n=4$ ).

The overall process is shown below(Fig.1):

1. Perform a new coronavirus fast test (Fastv) on all data to determine whether it is negative or positive. Compare the virus database to find other co-infected viruses. Compare the bacterial database and annotate the species of the bacteria.

2. Compare to the original data (SortmeRNA) ribosomal database. Split the data into other and rRNA parts.

3. Compare the other part of the reads to hg19. Perform differential analysis of human expressed genes.

4. Compare the other part of the reads with SARS-CoV-2 (NC_045512.2). Analyze virus coverage, depth, mutation, and L/S typing.

5. After excluding the human and SARS-CoV-2 sequences from the other part:

1) Assemble of the 8 throat swabs and 6 stool data (Metaspades). And Remove redundant sequences (Cd-hit). Assess the effect of assembly (Quast).

2) Take the above assembly result as the reference sequence, and do quantitative statistics for all samples (Salmon).

3) Analysis of differences between the negative and positive groups.

4) Perform species and function analysis (Humann3).

\section{Statistical analysis}

Continuous variables are expressed as median (IQR) and compared using the Mann-Whitney U test. Categorical variables are expressed as numbers (\%) and compared by the chi-square test or Fisher's exact test. A two-sided a of less than 0.05 was considered statistically significant.

\section{Results}

SARS-CoV-2 and Influenza $A V B$ virus infection trends during the epidemic 
By Feb 1, 2020, 649 fever patients were doubted as SARS-CoV-2 infection in Hunan. The patients were aged from 2 to 65 , and the median age of the patients was 46 years (IQR 29.25-45.5). During the COVID epidemic, all the fever patients admitted to the hospital were tested for the SARS-CoV-2 and Flu A/B nucleic acid, using the kits of bioPerfectus and Sansure biotech respectively. Among fever patients, 3.96\% $(29 / 733)$ of patients are infected with the Influenza A virus and $5.46 \%(40 / 733)$ are infected with the Influenza $B$ virus. We found that patients with SARS-CoV-2 do not have co-infections of A and $B$. The number of newly diagnosed patients with SARS-CoV-2 accounts for only $2.05 \%$ of all fever patients (Tab. 1). The number of COVID-19 confirmed cases in these six days (Jan 23th, Jan 26, Jan 28, Jan 29, Jan 31, and Feb 1st) is relatively stable. The number of new patients with fever infected with Influenza $A / B$ virus fluctuates greatly and is significantly higher than the number of COVID-19 patients $(P<0.01)$ (Fig.2).

\section{Changes in Influenza $A / B$ virus before, during and after the COVID-19 epidemic}

Before the epidemic, most patients with fever would only be tested for Influenza $A / B$ virus antigen. If the patient has a negative antigen test but is highly suspected of being infected with Influenza $A / B$ virus or if an antigen-positive patient wants to seek further diagnosis, the doctor will recommend the patient to take a nucleic acid test. Therefore, the results of the Influenza $A / B$ virus tests of patients before the epidemic originated from the antigen gold labeling method. However, during the epidemic, to ensure high detection efficiency and timely treatment of COVID-19 patients, all fever patients in this teaching hospital detected Influenza $A / B$ virus and SARS-CoV-2 nucleic acid. The positive rate of each period is shown in Tab.2. The detection rate of Influenza $A / B$ virus in fever patients from 2019.8 .1 to 8.16 is $8.62 \%(151 / 1751)$ via colloidal gold immunochromatography. The nucleic acid test result is $7.48 \%(8 / 107)$ similar to that of the immunoassay. During the epidemic (2020.1-2020.2), the infection rate of the Influenza A/B virus increased to $12.55 \%$ (92/733), showing the same trend as the COVID-19. However, After the epidemic, the detection rate of COVID-19 among fever patients in this teaching hospital dropped to zero and the infection rate of the Influenza $A / B$ virus became extremely low (0.00\%)(Fig.3).

\section{Infections of pathogens in fever patients}

We tested ten respiratory pathogen nucleic acid detection for patients. The test results are as follows (Tab.3): The most common pathogen at onset of illness was Respiratory syncytial virus (RSV) [16 (2.47\%) of 649 patients]. Influenza A(FluA) [9 (1.39\%) of 649 patients], Influenza B(FluB) [14 (2.16\%) of 649 patients], M.Pneumonia(MP) [11 (2.47\%) of 649 patients], Adenovirus(ADV) [10 (1.54\%) of 649 patients], Parainfluenza virus I (PIV1) [0 (0.00\%) of 649 patients], Parainfluenza virus II (PIV2) [1 (0.15\%) of 649 patients], Parainfluenza virus III (PIV3) [0 (0.00\%) of 649 patients], Coronavirus virus 229E (HCoV229E) [5 (0.77\%) of 649 patients], Human metapneumovirus (HPMV) [13 $(2.00 \%)$ of 649 patients], Human Coronavirus virus OC43 (HCoV-OC43) [1 (0.15\%) of 649 patients], Human Boca virus (HBoV) [0 (0.00\%) of 649 patients]. The proportion of patients with SARS-CoV-2 among fever patients is [15/733 (2.05\%)]. The other febrile pathogens rate of fever patients is higher than patients with SARS-CoV-2 $(P<0.01)$ (Fig. 4).

\section{Basic statistics of bioinformatics analysis data}


Quickly check the SARS-CoV-2 virus. The mean and depth of SARS-CoV-2 coverage of positive samples were $98.03 \%$ (44.80\%-99.87\%) and 12.9X (2.8X-167.8X). The statistics are as follows (Tab. 4):

\section{Analysis of variation and intra-host variation}

Use samtools mpileup and in-house scripts to analyze SARS-CoV-2 mutations. Extract all mutation sites with a frequency above $90 \%$ and the site depth $>=10 \mathrm{X}$. A total of 18 loci were detected from 9 samples. 209001889R and 209001909R share 2 identical mutations (27925, 28377). 209001897R and 209001903R share the C28854T mutation (Tab.5).

Extract all the sites with a frequency of more than $5 \%$ and the genotype depth of the mutation $>5 X$, and remove the sites with a mutation frequency of more than $95 \%$. A total of 42 Intra-host mutations (intrahost mutations) were obtained, which were sporadic. The same mutation was not found in any two samples. For details, please see " Supplementary Table 1".

Extracting the genotypes at positions 8782 and 28144 in all samples, 5 samples can distinguish the L/S type, including $4 \mathrm{~S}$ type and $1 \mathrm{~L}$ type (Tab.6).

\section{SARS-Cov-2 and human genome integration analysis}

For the sample "209001902R" with the highest virus depth, the reads that were not completely aligned to humans and that were not completely aligned to SARS-Cov-2 were re-analyzed, and no reads that reliably supported fusion were found (Supplementary Table 2).

\section{Analysis of mixed infection with other pathogens}

4(25\%) positive samples were combined with Human adenovirus infection. 209001894R was Human respiratory syncytial virus B infection (Supplementary Table 3).

\section{Metatranscriptome analysis}

The data of the 8 throat swabs and 6 stool samples were spaded and de-redundant (cd-hit) to obtain the gene set. And use QUAST to evaluate the assembly effect. Compare the reference gene set (throat swabs and stool separately) to all samples, and calculate the expression level. Use "edgeR" to do DEGs analysis on the negative and positive groups. The results are shown in the form " Supplementary Table 4". The volcano plot (ggplot2) of the differential expression of transcripts of swab and stool in negative and positive samples is shown in the Figure 5.

Metaphlan was used to analyze the origin of mRNA sequence species. Calculate the Shannon, Simpson, and inverse Simpson indexes ( $R$ package vegan) at the species and genus levels. Calculate t-test, Wilcox test value (python3 scipy). Calculate the kind of horizontal unifrac distance (R package rbiom). Draw PCoA diagrams (ape, ggplot2). See Supplementary Table 4 form "Diversity". The following Fig. 6 is a PCoA diagram made by unifrac distance. Humann3 is used to make functional annotations on the part of the macro transcript data, and the difference between the positive and negative two groups is calculated. The 
pathways with Wilcox-test p-value $<0.05$, t-test p-value $<0.05$ and Equal_Vari_P $>0.05$ (levene test for homogeneity of variance) were screened out (Tab.7 and Supplementary Table 5).

For the rRNA part, use Kraken2 to do taxonomy on the data, and calculate the Shannon, Simpson, and inverse Simpson indices. There was no significant difference in species and genus levels (Wilcox-test, $p=0.1824, p=0.1654)$.

\section{Discussion}

During the epidemic, although we must pay attention to the spread of SARS-CoV-2 and medical control, appropriate infection control measures must be also implemented to prevent and control possible infections of other respiratory pathogens. Clinicians should not ignore that common respiratory pathogens cause fever or even co-infection with SARS-CoV- $2^{21}$. Therefore, this study aims to better understand the fever symptoms of patients caused by common respiratory pathogens during the COVID19 epidemic.

The co-infection of Influenza $A / B$ virus at COVID-19 diagnosis is uncommon. Most patients have a fever caused by common respiratory pathogens such as Influenza $A$ virus (FluA), Influenza B virus (FluB), M.Pneumonia(MP), Adenovirus (ADV), Respiratory syncytial virus (RSV), Parainfluenza virus I(PIV1), Parainfluenza virus II (PIV2), Parainfluenza virus III(PIV3), Coronavirus virus 229E (HCoV-229E), Human metapneumovirus (HPMV), Human Coronavirus virus OC43 (HCoV-OC43), Human Boca virus (HBoV). These findings are quite different when compared with those of other viral pandemics. As it relates to hospitalised patients with COVID-19, such findings could prove essential in defining the role of empiric antimicrobial therapy or stewardship strategies. At present, most doctors for patients with confirmed SARS-CoV-2 will use broad-spectrum antibacterial and antiviral treatment. 99 COVID-19 patients in Wuhan, China reported that $71 \%, 76 \%$, and $15 \%$ used antibiotics, antiviral agents, and antifungal agents $^{22}$. For confirmed patients, clinicians can give patients empirical broad-spectrum antibacterial treatment according to relevant guidelines ${ }^{23}$. But for patients with undiagnosed fever to avoid the abuse of antibiotics, we should use drugs carefully. According to the data shown in our research, infectious doctors still have to pay great attention to the infection of common respiratory pathogens in patients with fever, and can give patients ordinary anti-cold drugs.

In order to further explore the impact of SARS-CoV-2 infection on the host and co-infection with other respiratory pathogens. We conducted a rapid test for the new coronavirus (fastv) on 26 fever samples. Comparing virus library and bacterial library for species annotation. We found that the mutation after SARS-CoV-2 infection was sporadic, and the same mutation was not found in any two samples. Due to the defects of sample quality, only 5 samples can distinguish L/S type, of which 4 are $S$ type and 1 is $L$ type. This part of the data indicates that the SARS-CoV-2 detected in patients with covid-19 in low-risk

areas in China has a small trend of variation and most of them are low-virulence S-types ${ }^{24,25}$. And the study found that there is no integration phenomenon between SARS-Cov-2 and the human genome. The study found that SARS-CoV-2 positive samples will also be infected with other viruses, especially 
adenovirus. Therefore, it is necessary to use antiviral preventive drugs for fever patients during the epidemic $^{26}$. Macrotranscript analysis showed that there was no significant difference in the species and genus levels of pathogens between covid-19 patients and other fever patients. This finding proves that SARS-Cov-2 infection does not cause significant changes in the human microbial population. In addition, the main pathways that affect human metabolism after SARS-Cov-2 infection are the Calvin-BensonBassham cycle, pyrimidine deoxyribonucleotides de novo biosynthesis I and D-galactose degradation V (Leloir pathway) and other common aerobic respiration and nucleic acid metabolism pathways. This also further confirmed the positive regulation of SARS-Cov-2 on human oxidative stress and inflammatory storm. Clinicians need to pay attention to the co-infection of covid-19 patients with pathogens, but also to pay attention to the patient's blood gas, electrolyte levels, and peripheral blood immune factor levels ${ }^{27}$.

\section{Conclusion}

During the COVID-19 epidemic, due to the strengthening of the Chinese government, medical institutions, and public protection measures, the number of patients with Influenza $A / B$ virus during the same period has been greatly reduced. In the face of fever patients, clinicians still need to pay more attention to infections of common respiratory pathogens in addition to t SARS-CoV-2. China's public health measures to stop the spread of the epidemic have proven effective.

\section{Abbreviations}




\begin{tabular}{|ll|}
\hline COVID-19 & Corona Virus Disease 2019 \\
\hline SARS-CoV-2 & Severe acute respiratory syndrome coronavirus 2 \\
\hline MERS & the Middle East respiratory syndrome \\
\hline hMPV & Human metapneumovirus \\
\hline hBoV & Human Boca virus \\
\hline hCoV & Human coronavirus \\
\hline RSV & Respiratory syncytial virus \\
\hline MP & Mycoplasma pneumoniae \\
\hline PCR & Polymerase Chain Reaction \\
\hline FluA & Influenza A \\
\hline FluB & Influenza B \\
\hline MP & M.Pneumonia \\
\hline ADV & Adenovirus \\
\hline PIV & Parainfluenza virus \\
\hline HCoV-229E & Coronavirus virus 229E \\
\hline HCoV-OC43 & Human Coronavirus virus OC43 \\
\hline
\end{tabular}

\section{Declarations}

\section{Ethical Approval and Consent to participate}

Not Applicable

\section{Consent for publication}

All authors agree to publish.

\section{Availability of data and materials}

The data that support the findings of this study are available from the corresponding author upon reasonable request.

\section{Competing interests}

All the authors have no conflict of interest.

\section{Funding}


This work was supported by grants from the Hunan Provincial Natural Science Foundation of China (Grant No. 2019JJ40426), the Hunan Provincial Natural Science Foundation of China (Grant No. 2019JJ40418), the Hunan Provincial Clinical Medical Technology Innovation Guide Project (Grant No. 2017SK50122), the Fundamental Research Funds for the Central Universities of Central South University (Grant NO. 2019zzts806)

\section{Authors' contributions}

All authors have made substantial contributions to conception and design, or acquisition of data, or analysis and interpretation of data; and been involved in drafting the manuscript or revising it critically for important intellectual content, and participated sufficiently in the work to take public responsibility for appropriate portions of the content, and agreed to be accountable for all aspects of the work.

\section{Acknowledgements}

All authors would like to thank agent manufacturers that provided the real-time RT-PCR kits and instruments for SARS-CoV-2 detection in this study.

\section{References}

1. Wu Z, McGoogan JM. Characteristics of and Important Lessons From the Coronavirus Disease 2019 (COVID-19) Outbreak in China: Summary of a Report of 72314 Cases From the Chinese Center for Disease Control and Prevention. Jama. 2020.

2. Zhu N, Zhang D, Wang W, et al. A Novel Coronavirus from Patients with Pneumonia in China, 2019. The New England journal of medicine. 2020;382(8):727-733.

3. Pan X, Chen D, Xia Y, et al. Asymptomatic cases in a family cluster with SARS-CoV-2 infection. The Lancet Infectious diseases. 2020.

4. Wang N, Li SY, Yang XL, et al. Serological Evidence of Bat SARS-Related Coronavirus Infection in Humans, China. Virologica Sinica. 2018;33(1):104-107.

5. Li Q, Guan X, Wu P, et al. Early Transmission Dynamics in Wuhan, China, of Novel CoronavirusInfected Pneumonia. The New England journal of medicine. 2020.

6. Huang C, Wang Y, Li X, et al. Clinical features of patients infected with 2019 novel coronavirus in Wuhan, China. Lancet (London, England). 2020;395(10223):497-506.

7. Thompson R. Pandemic potential of 2019-nCoV. The Lancet Infectious diseases. 2020.

8. Garcia-Vidal C, Sanjuan G, Moreno-García E, et al. Incidence of co-infections and superinfections in hospitalised patients with COVID-19: a retrospective cohort study. 2020.

9. Yin S, Peng Y, Ren Y, et al. The implications of preliminary screening and diagnosis: Clinical characteristics of 33 mild patients with SARS-CoV-2 infection in Hunan, China. 2020;128:104397.

10. Standl F, Jöckel K, Brune B, Schmidt B, Stang AJTLId. Comparing SARS-CoV-2 with SARS-CoV and influenza pandemics. 2020. 
11. Pawełczyk M, Kowalski MJCa, reports a. The Role of Human Parainfluenza Virus Infections in the Immunopathology of the Respiratory Tract. 2017;17(3):16.

12. Marinari L, Danny M, Simpson S, Schmitt J, Miller WJEjor. Lower Respiratory Tract Infection with Human Metapneumovirus: Chest CT Imaging Features and Comparison with Other Viruses. 2020;128:108988.

13. Madi N, Al-Adwani AJJomm. Human bocavirus (HBoV) in Kuwait: molecular epidemiology and clinical outcome of the virus among patients with respiratory diseases. 2020;69(7):1005-1012.

14. Zou L, Yi L, Yu J, et al. Adenovirus infection in children hospitalized with pneumonia in Guangzhou, China. 2020.

15. Rucinski S, Binnicker M, Thomas A, Patel RJMCp. Seasonality of Coronavirus 229E, HKU1, NL63, and OC43 From 2014 to 2020. 2020;95(8):1701-1703.

16. Öner D, Drysdale S, McPherson C, et al. Biomarkers for Disease Severity in Children Infected With Respiratory Syncytial Virus: A Systematic Literature Review. 2020.

17. Gu L, Liu W, Lai G, et al. [Epidemiological investigation and analysis of an outbreak of mycoplasma pneumoniae pneumonia in adult]. 2018;98(46):3784-3788.

18. Zheng $B$, Wang H, Yu CJPp. An increasing public health burden arising from children infected with SARS-CoV2: a systematic review and meta-analysis. 2020.

19. Song G, Liang G, Liu WJM. Fungal Co-infections Associated with Global COVID-19 Pandemic: A Clinical and Diagnostic Perspective from China. 2020.

20. Zheng Q, Lu Y, Lure F, Jaeger S, Lu PJJoX-rs, technology. Clinical and radiological features of novel coronavirus pneumonia. 2020;28(3):391-404.

21. Oliva A, Siccardi G, Migliarini A, et al. Co-infection of SARS-CoV-2 with Chlamydia or Mycoplasma pneumoniae: a case series and review of the literature. 2020.

22. Chen N, Zhou M, Dong X, et al. Epidemiological and clinical characteristics of 99 cases of 2019 novel coronavirus pneumonia in Wuhan, China: a descriptive study. 2020;395(10223):507-513.

23. Guan W, Ni Z, Hu Y, et al. Clinical Characteristics of Coronavirus Disease 2019 in China. 2020;382(18):1708-1720.

24. Cheng L, Han X, Zhu Z, Qi C, Wang P, Zhang XJBib. Functional alterations caused by mutations reflect evolutionary trends of SARS-CoV-2. 2021.

25. Daniloski Z, Jordan T, IImain J, et al. The Spike D614G mutation increases SARS-CoV-2 infection of multiple human cell types. 2021;10.

26. Babiker A, Bradley H, Stittleburg V, et al. Metagenomic Sequencing To Detect Respiratory Viruses in Persons under Investigation for COVID-19. 2020;59(1).

27. Nascimento Conde J, Schutt W, Gorbunova E, Mackow EJm. Recombinant ACE2 Expression Is Required for SARS-CoV-2 To Infect Primary Human Endothelial Cells and Induce Inflammatory and Procoagulative Responses. 2020;11(6). 


\section{Tables}

Due to technical limitations, tables are only available as a download in the Supplemental Files section.

Figures

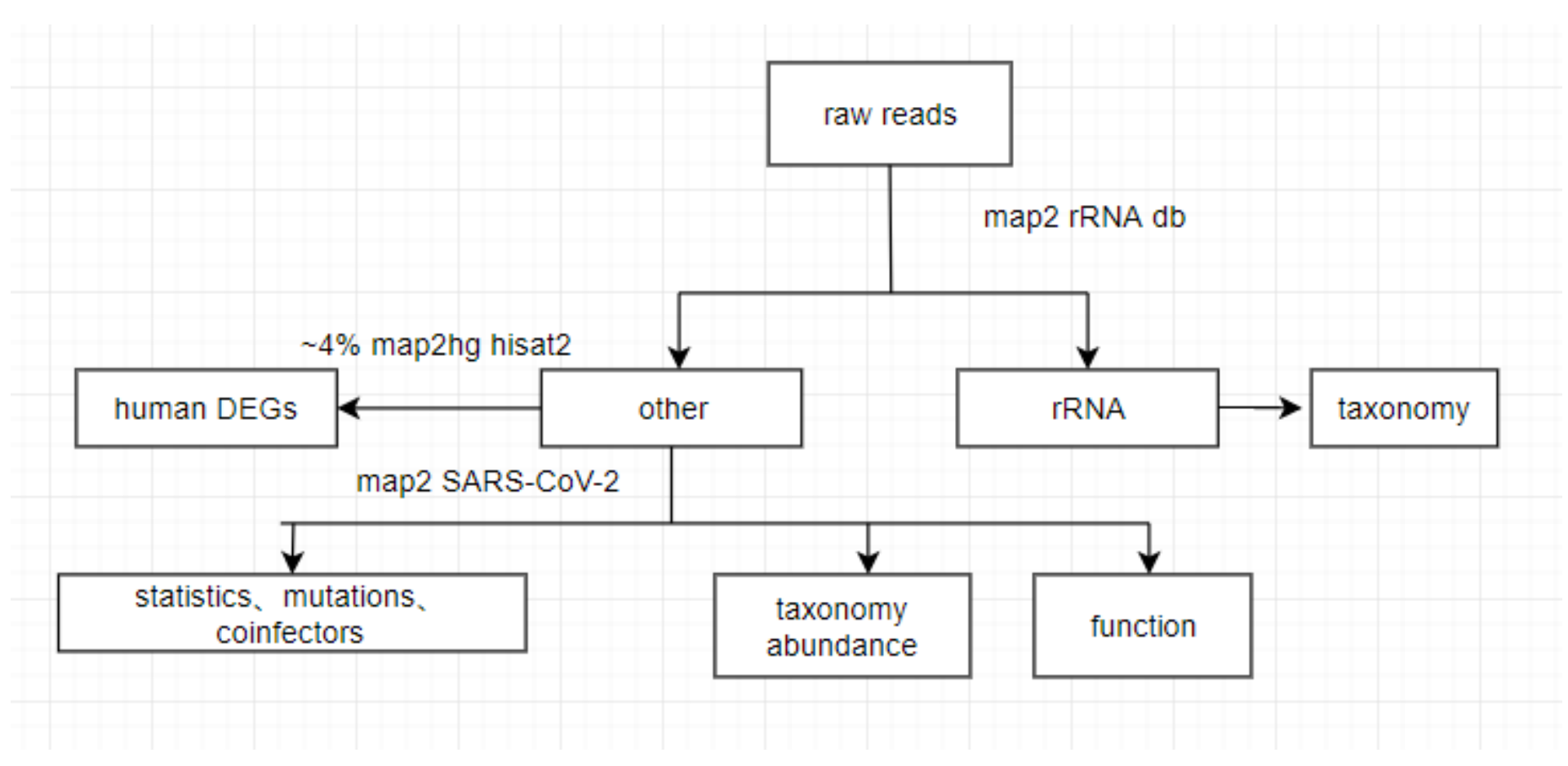

Figure 1

Bioinformatics analysis process 


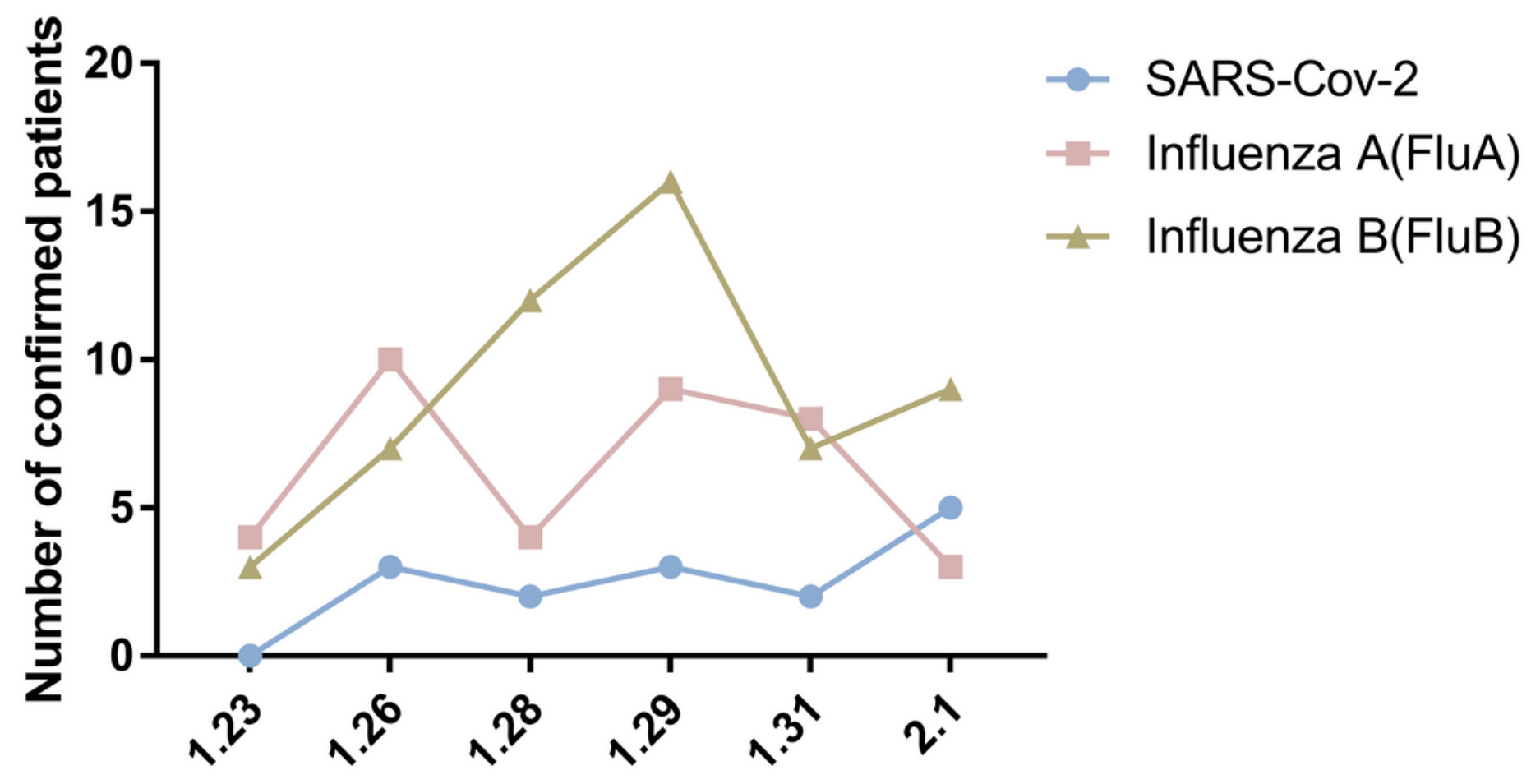

Figure 2

SARS-CoV-2 and Influenza A/B virus infection trends during the epidemic.

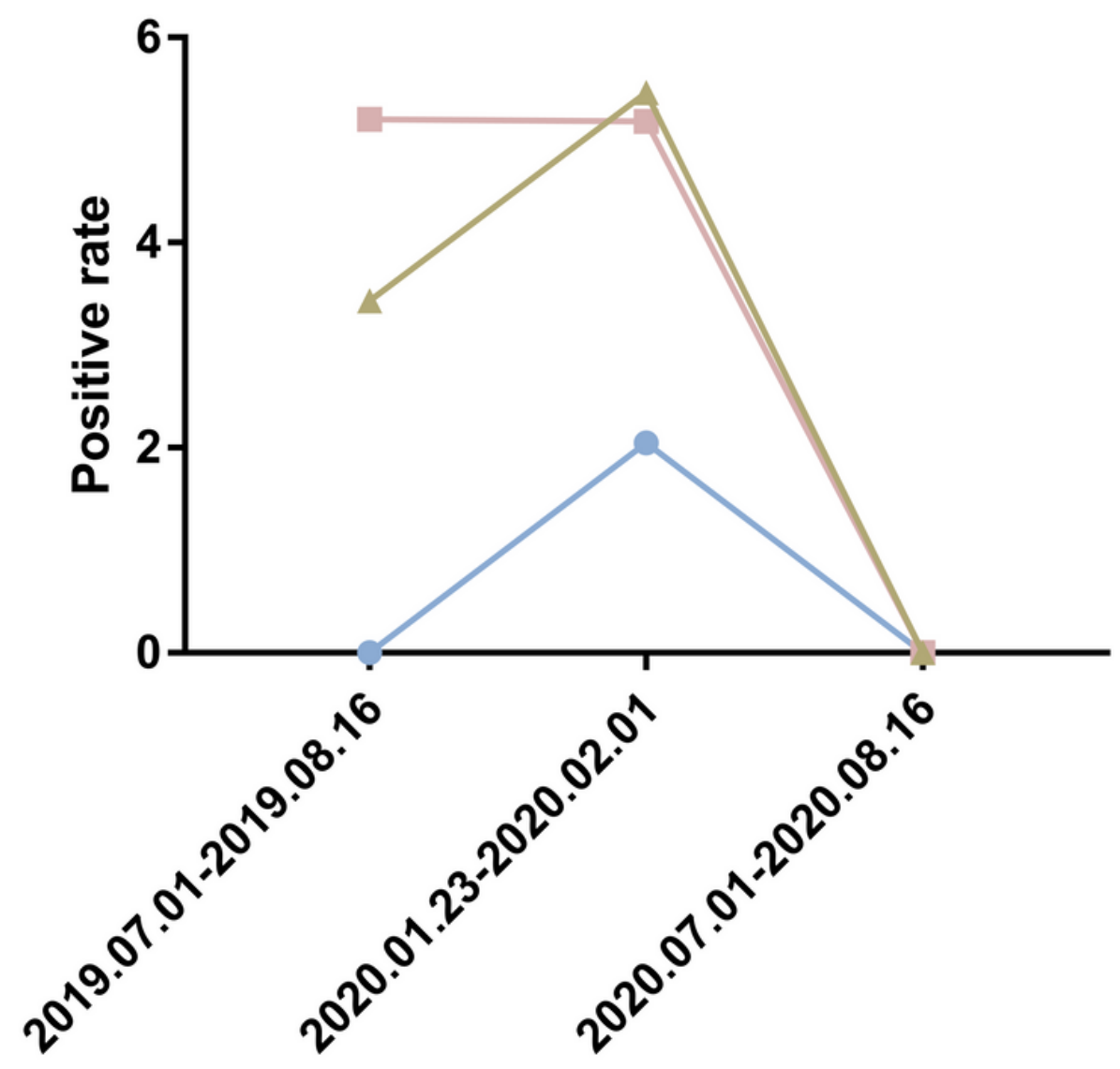

$\rightarrow$ SARS-Cov-2

-- Influenza A(FluA)

$-\quad$ Influenza B(FluB) 
Figure 3

Changes in Influenza A/B virus before, during, and after the COVID-19 epidemic. 2020.1.23-2.1: Jan 23th, Jan 26, Jan 28, Jan 29, Jan 31, and Feb 1st.
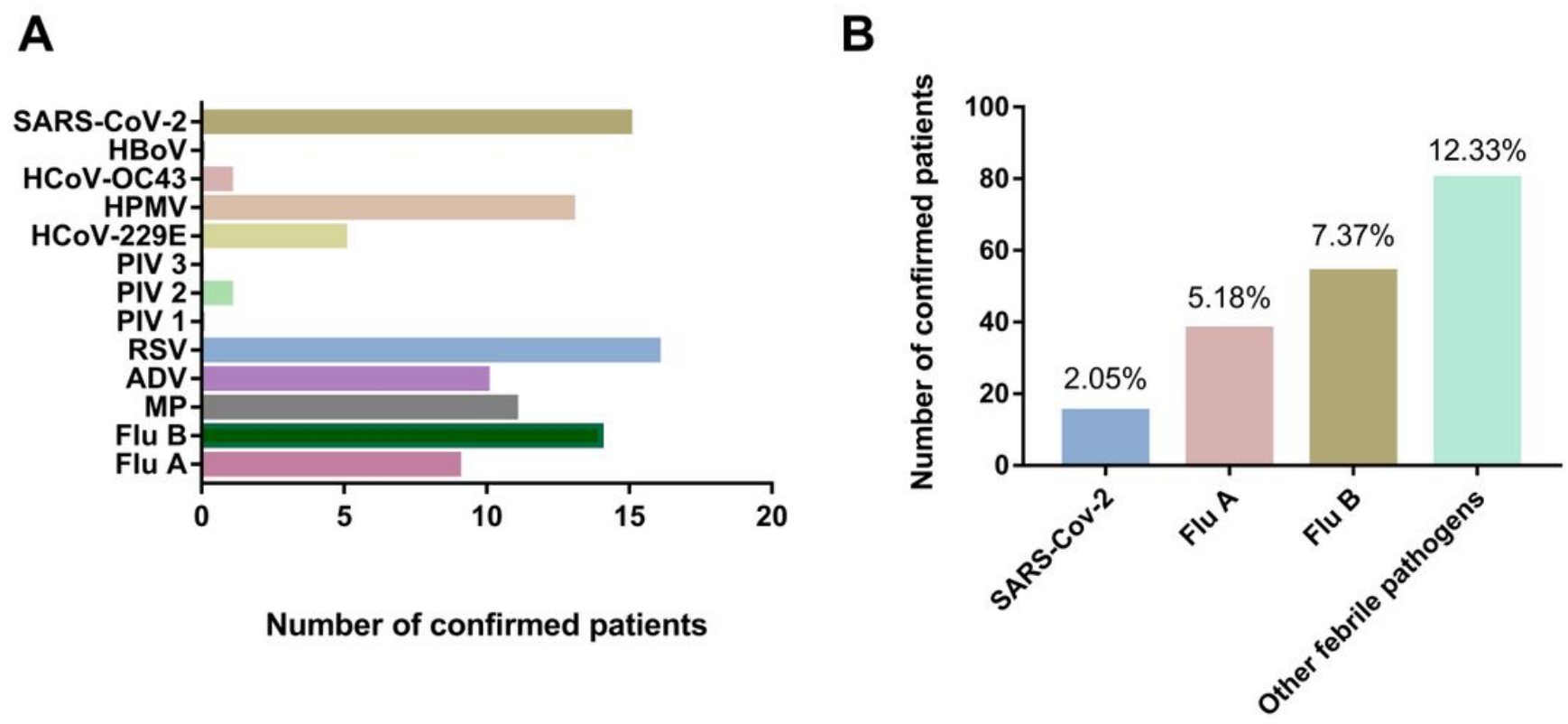

\section{Figure 4}

Infections of other pathogens in fever patients without SARS-CoV-2.

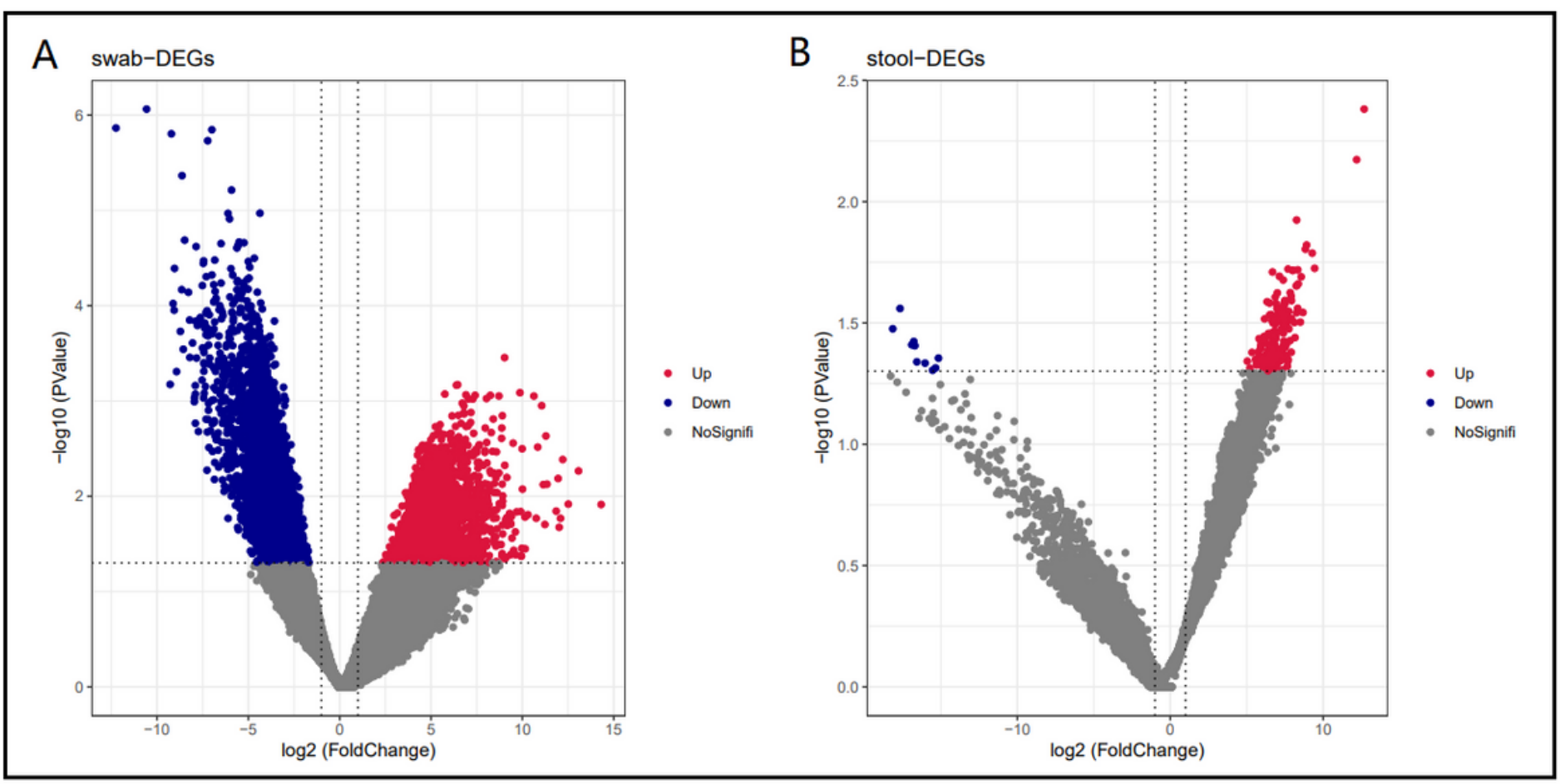


Figure 5

(A)The volcano plot of the differential expression of transcripts of swab in negative and positive samples. (B)The volcano plot of the differential expression of transcripts of stool in negative and positive samples. For differential transcripts, please see the form "throat swab-DEGs" and "stool-DEGs" in Supplementary Table 4.

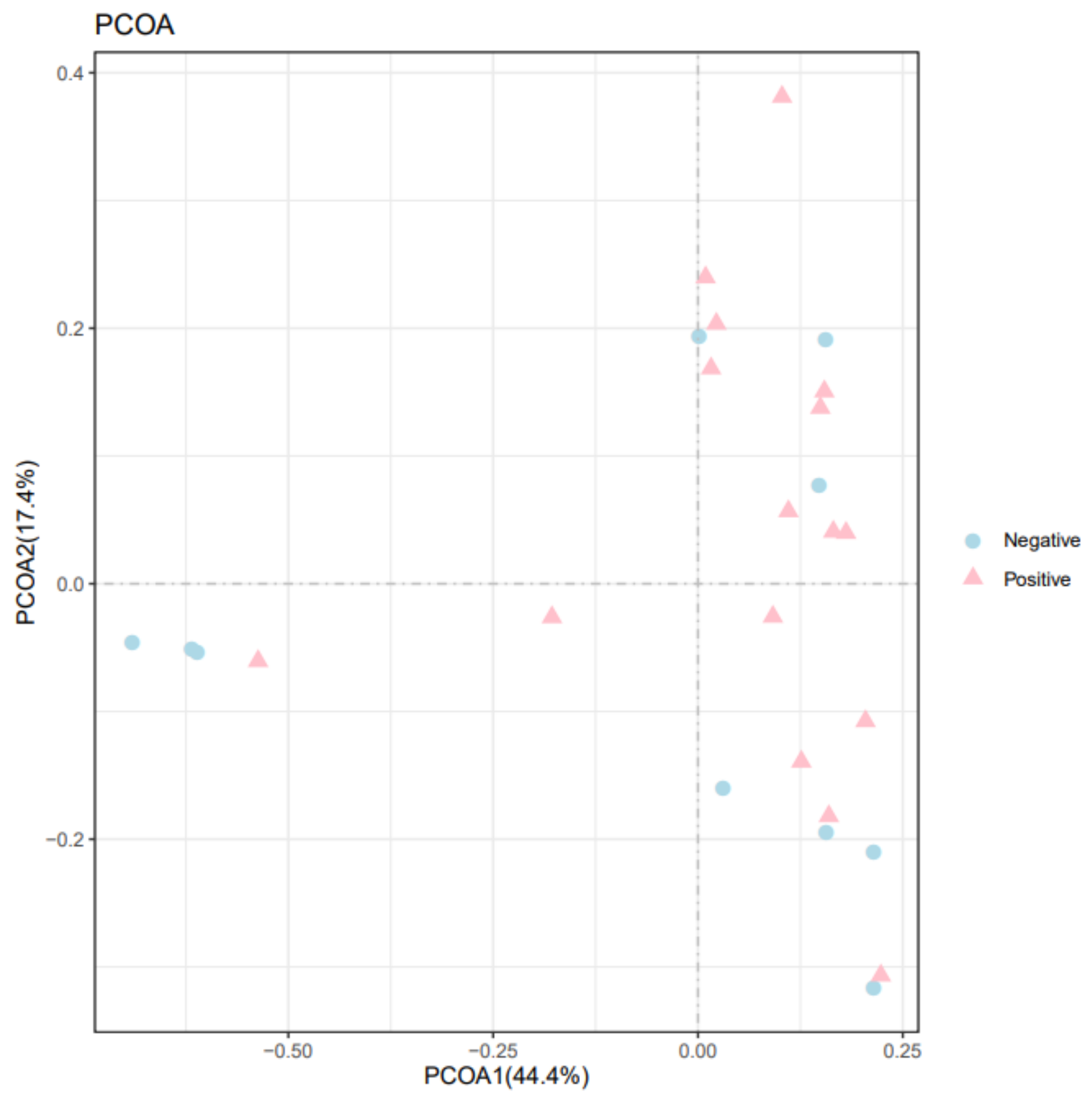

Figure 6

PCoA diagram made by unifrac distance

Supplementary Files 
This is a list of supplementary files associated with this preprint. Click to download.

- SupplementaryTable1.xlsx

- SupplementaryTable2.xlsx

- SupplementaryTable3.xlsx

- SupplementaryTable4.xlsx

- SupplementaryTable5.xlsx

- Tab.1.xlsx

- Tab.2.xIsx

- Tab.3.xIsx

- Tab.4.xlsx

- Tab.5.xlsx

- Tab.6.xlsx

- Tab.7.xIsx 